\title{
Dynamics of electron beam ablation of silicon dioxide measured by dye laser resonance absorption photography
}

\author{
S. D. Kovaleski, R. M. Gilgenbach, ${ }^{\text {a) }}$ L. K. Ang, and Y. Y. Lau \\ Intense Energy Beam Interaction Laboratory, Department of Nuclear Engineering and Radiological \\ Sciences, The University of Michigan, Ann Arbor, Michigan 48109-2104
}

(Received 16 July 1998; accepted for publication 3 September 1998)

\begin{abstract}
The dynamics of electron beam ablation plumes have been characterized through the application of dye laser resonance absorption photography. The ablation of fused silica by a channelspark electron beam was studied by probing the near-ground state, $3 p^{2}{ }^{1} D-4 s^{1} P^{0}$ neutral $\mathrm{Si}$ transition at $288.158 \mathrm{~nm}$. Necessary background gases $\left(\mathrm{Ar}\right.$ or $\mathrm{N}_{2}$ ) were tested at pressures of 15 or 30 mTorr. A two-lobed, Si atom plume shape was discovered that is hydrodynamically more complex than laser ablation plumes. These plumes merge into a single-lobed plume at about $400 \mathrm{~ns}$ after the e-beam current pulse rise. Plume front expansion velocities of Si atoms were measured at nearly $1 \mathrm{~cm} / \mu \mathrm{s}$, and are comparable to the expansion of laser ablated metal atom plumes with laser fluences of a few $\mathrm{J} / \mathrm{cm}^{2}$. (C) 1998 American Institute of Physics. [S0003-6951(98)02444-9]
\end{abstract}

Energy beam ablative deposition of thin films has been demonstrated to be an important technique for metals, insulators, and superconductors. ${ }^{1,2}$ Most ablation experiments have utilized ultraviolet excimer lasers $(\mathrm{KrF}, \mathrm{XeCl})$ as the energy source. Recently, electron beam sources with high current (kA) and moderate voltage (10-15 kV) have become available, enabling electron beam driven ablative deposition of thin films. ${ }^{3}$ Electron beam ablation plasmas have been studied through the optical emission of plume neutrals and ions. ${ }^{4,5}$ The potential advantages of electron beam ablation are higher energy efficiency, ability to ablate optically transparent materials (e.g., $\mathrm{SiO}_{2}$ ), and lower capital costs. An environmental advantage of e-beam ablative deposition of $\mathrm{SiO}_{2}$ is that it does not require the use of toxic silane gas.

In this letter, we present the first direct measurements of channelspark electron beam ablated $\mathrm{Si}$ neutral atom plume dynamics by dye laser resonance absorption photography (DLRAP). These measurements probe nonemissive plume constituent dynamics during and following the e-beam ablation process. The experiments studied the ablation of fused silica, chosen for its importance in the production of semiconductor microelectronics and because of its transparency to ultraviolet laser light. Background gases included Ar or $\mathrm{N}_{2}$ at pressures of 15 or of $30 \mathrm{mTorr}$ for each.

A schematic drawing of the DLRAP diagnostic ${ }^{6}$ of the electron beam ablation experiment is detailed in Fig. 1. The ablation-driving electron beam source is a channelspark developed at Kernforschungszentrum Karlsruhe. ${ }^{3}$ The channelspark has the following parameters: ${ }^{5}$ negative $15-20 \mathrm{kV}$ charging potential, $1500 \mathrm{~A}$ beam current, 7.5-30 mTorr of background gas pressure for ion focusing (either Ar or $\mathrm{N}_{2}$ for this experiment), pulse energy of about $1 \mathrm{~J}$, incident fluence less than $50 \mathrm{~J} / \mathrm{cm}^{2}$, spot size $0.02 \mathrm{~cm}^{2}$, and pulse length of 200 ns. The authors have installed a triggered spark-gap switch for command triggering the channelspark. The channelspark electron beam impacts a fused silica target whose surface normal is canted $45^{\circ}$ from the electron beam axis.

a)Electronic mail: rongilg@umich.edu
The ablation plume expands along the target surface normal. Note that for insulating targets the return current must flow through the e-beam produced $\mathrm{Ar}$ or $\mathrm{N}_{2}$ plasma. DLRAP is performed using a dye laser pumped by a $\mathrm{XeCl}$ excimer laser. The dye laser has a pulse length of $20 \mathrm{~ns}$ and a linewidth of $0.2 \mathrm{~cm}^{-1}$. Frequency doubled laser light (from Coumarin 153 dye) is absorbed by the near-ground state, neutral Si $3 p^{2}{ }^{1} D-4 s{ }^{1} P^{0}$ transition at $288.158 \mathrm{~nm}$. The dye laser beam is expanded and recollimated through a telescope and passed perpendicular to the ablation plume and electron beam. The DLRAP camera is set up in an open shutter arrangement; a lens focuses the diagnostic beam through an adjustable iris, to reduce plasma light from the ablation event, and images the absorption on high-speed photographic

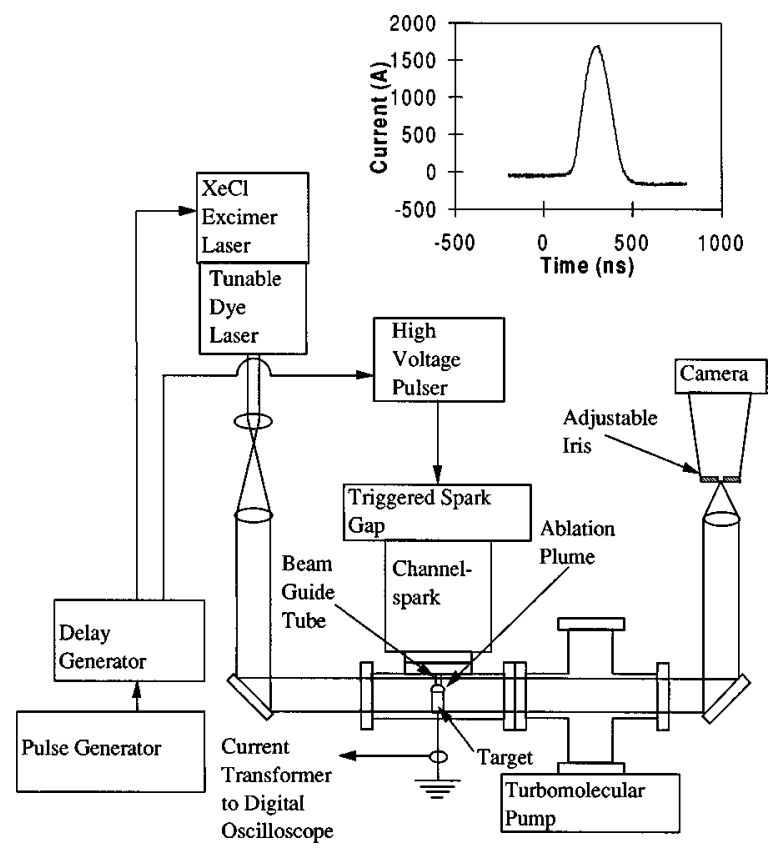

FIG. 1. Schematic drawing of electron beam ablation experiment with dye laser resonance absorption photography diagnostic. The inset shows a typical channelspark current pulse, measured at a metal target, for a low background gas pressure case. 


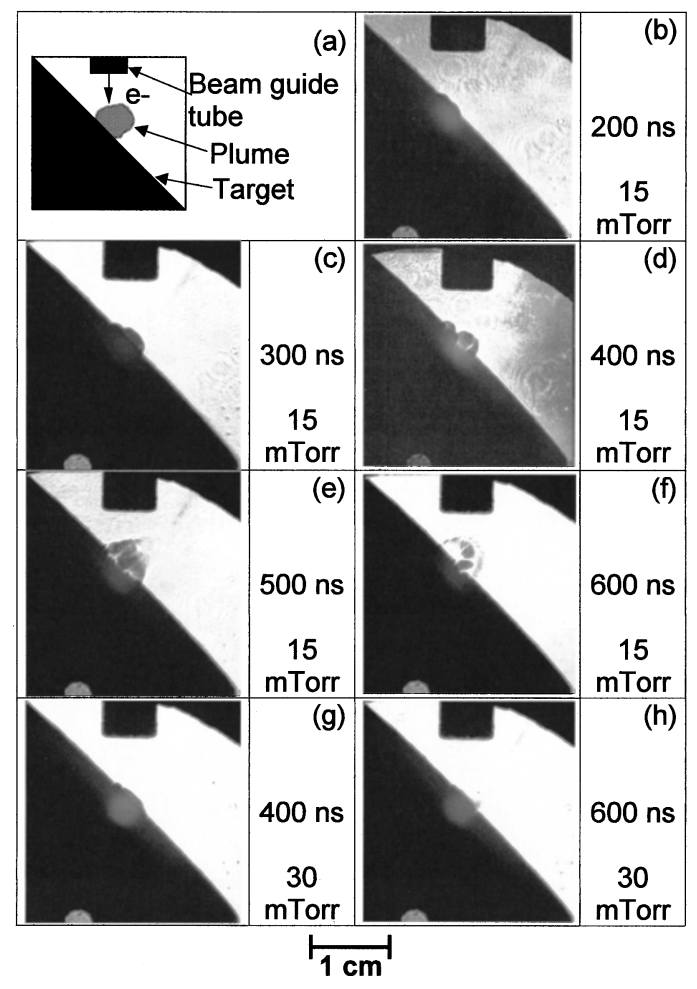

FIG. 2. DLRAP photos of Si neutral atom plumes ablated in argon gas. (a) Schematic drawing of DLRAP pictures. (b)-(f) Photos at different delay times between the time when the e-beam current has risen to $10 \%$ of its maximum and the dye laser pulse, for argon gas pressure of 15 mTorr. (g),(h) Photos at different delay times for argon gas pressure of $30 \mathrm{mTorr}$. Photographs digitally background subtracted to remove optical noise due to dust and optical imperfections and processed to improve contrast.

film (Polaroid type 667). Photographs have been scanned into a personal computer for adjustment of contrast and background subtraction. A digital delay generator controls the relative delay between the channelspark electron beam and the dye laser pulse.

DLRAP photographs of the electron beam ablation of fused silica are shown in Fig. 2. Each photograph corresponds to a single dye laser pulse that is delayed by a specified time following the channelspark electron beam pulse. The delay times are measured relative to when the current, measured at the target, first reaches $10 \%$ of its maximum. As the photographic equipment is in an open shutter arrangement, the shuttering time is equal to the pulse width of the dye laser, $20 \mathrm{~ns}$. Near the end of the e-beam pulse [Fig. 2(b)] at $200 \mathrm{~ns}$, the absorption of the dye laser beam by the ablated Si neutral atoms has become strong enough for photographic detection. The initial plume is quite wide, $4.5 \mathrm{~mm}$, with what appears to be a double-lobed hydrodynamic structure. Figures 2(b), 2(c), and 2(d), corresponding to times of 200, 300, and $400 \mathrm{~ns}$ after the initiation of the electron beam all exhibit this double-lobed plume structure. At 500 ns, shown in Fig. 2(e), the two lobes of the Si neutral atom plume have hydrodynamically merged into a conical-shaped plume. This conical shape then persists for the remainder of the ablation event, although nonuniform plume shapes suggest possible plume turbulence. DLRAP photographs at $600 \mathrm{~ns}$ [Fig. 2(f)], $700 \mathrm{~ns}$, and $800 \mathrm{~ns}$ (not shown), indicate that as the plume continues to expand, the absorption decreases steadily, due to the decreasing density of the expanding $\mathrm{Si}$ atoms. By 800

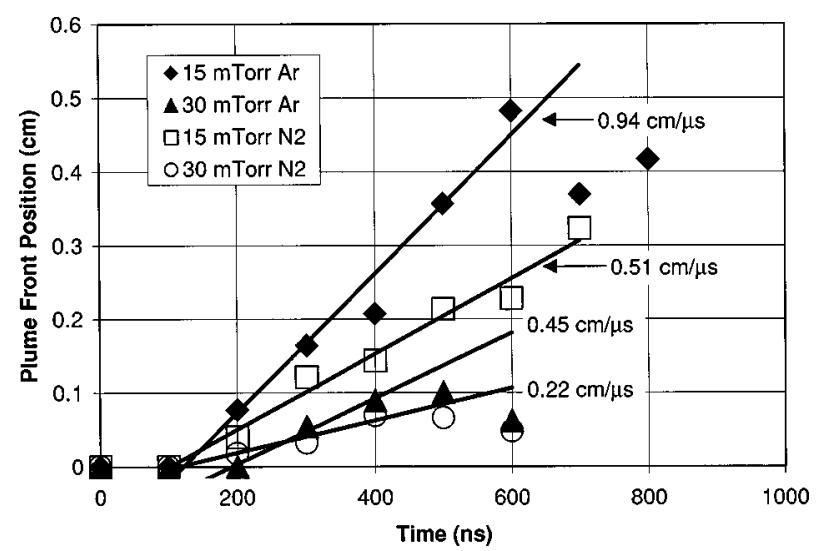

FIG. 3. Plots of Si neutral atom plume front position for $\mathrm{Ar}$ or $\mathrm{N}_{2}$ background gas at different pressures. Linear fit to data and expansion velocities are shown.

ns, absorption of the dye laser beam by the plume is nearly undetectable by DLRAP.

Figure 3 is a plot of the position of the Si neutral atom plume fronts with respect to time after initiation of the electron beam current pulse. Data are presented for two different background gases, $\mathrm{Ar}$ and $\mathrm{N}_{2}$, at two pressures for each gas, 15 and 30 mTorr. The plume front position is measured relative to the surface of the fused silica ablation target. For each gas, a linear least-squares fit was performed on the initial $\mathrm{Si}$ atom plume position-versus-time DLRAP data. This represents the plume trajectory, which is initially rising linearly, before the Si neutral atom density is too low to accurately measure the plume front. This fit is performed in order to determine an initial expansion velocity for the electron beam ablated $\mathrm{Si}$ atom plume. The highest expansion velocity, at $0.94 \mathrm{~cm} / \mu$ s was obtained for e-beam ablation in Ar background gas at 15 mTorr pressure. The expansion velocity of the plume generated in $\mathrm{N}_{2}$ at $15 \mathrm{mTorr}$ was about half as large as that for Ar, at $0.51 \mathrm{~cm} / \mu \mathrm{s}$. This velocity ratio of the plumes persists for both gases at higher pressures as well, with plumes generated in $\mathrm{Ar}$ at 30 mTorr expanding at 0.45 $\mathrm{cm} / \mu \mathrm{s}$ and those generated in $\mathrm{N}_{2}$ at 30 mTorr expanding at $0.22 \mathrm{~cm} / \mu \mathrm{s}$. These neutral atom plume expansion velocities correspond very well with those reported by Gilgenbach and Ventzek $^{6}$ and Lindley ${ }^{7}$ for $\mathrm{Al}$ neutral atom expansion after ablation by a $\mathrm{KrF}$ laser at laser fluences of $1.5-3 \mathrm{~J} / \mathrm{cm}^{2}$.

The silicon neutral atom plumes generated in $15 \mathrm{mTorr}$ in $\mathrm{N}_{2}$ are much like ablation plumes generated in vacuum; the plume expands fairly steadily and constantly through time. The same is largely true for plumes generated in 15 mTorr of Ar, except at late times, $700 \mathrm{~ns}$ and beyond. Si neutral line absorption at these times is very faint, however, and it is difficult to measure the position of the plume front due to the rapidly falling plume density. Plumes generated in $\mathrm{Ar}$ and $\mathrm{N}_{2}$ at 30 mTorr are very different compared to 15 mTorr. Figures $2(\mathrm{~g})$ and $2(\mathrm{~h})$ show that the plume is very dark through the entirety of its lifetime. The plumes are wide and flat, remaining close to the surface. Figure 3 shows that the edges of these plumes tend to erode at late times (500$600 \mathrm{~ns}$ ). The differences in expansion velocities between $\mathrm{Ar}$ and $\mathrm{N}_{2}$ and the difference in expansion velocity between 15 and $30 \mathrm{mT}$ Torr of either gas indicate a difference in the effective electron beam fluence delivered to the target surface. 
In conclusion, the dynamics of electron beam ablated $\mathrm{Si}$ neutral atom plumes have been determined through the application of dye laser resonance absorption photography. For ablation at background gas pressures of 15 mTorr Ar or of 15 mTorr $\mathrm{N}_{2}$, a two-lobed Si plume shape is evident in DLRAP photographs during the first $400 \mathrm{~ns}$; the two plumes hydrodynamically merge together after $400 \mathrm{~ns}$ and form a singlelobed ablation plume. The cause of the double-lobed Si atom plume is the subject of future investigations; a possible candidate includes instability of the high current beam. Ablation plumes persist for $800 \mathrm{~ns}$ before falling densities reduce the Si neutral atom absorption to undetectable levels. Si neutral atom plume front expansion velocities are on the order of 1 $\mathrm{cm} / \mu$ s comparable with the expansion of laser ablated $\mathrm{Al}$ neutral atom plumes with $\mathrm{KrF}$ laser fluences of a few $\mathrm{J} / \mathrm{cm}^{2}$. Ablation at background pressures of 30 mTorr $\mathrm{Ar}$ or $\mathrm{N}_{2}$ yields a slower moving plume that is stretched across the target, with less expansion energy in the direction perpendicular to the target.

Finally, we have extended this experiment to deposit a thin film from a $\mathrm{SiO}_{2}$ target, on a copper substrate, by some
5000 e-beam ablation pulses. Preliminary studies of these films indicate that they contain ablated particulate, suggesting a thermal melting and evaporation mechanism of ablation. Initial analysis of the film has indicated that oxygen is present and incorporated into the film.

This work was supported by National Science Foundation Grant No. CTS-9522282.

${ }^{1}$ Pulsed Laser Deposition of Thin Films, edited by D. G. Chrisey and G. K. Hubler (Wiley, New York, 1994).

${ }^{2}$ D. G. Geohegan and D. N. Mashburn, Appl. Phys. Lett. 55, 2345 (1989).

${ }^{3}$ G. Muller and C. Schultheiss, Proceedings of 10th International Conference On High Power Particle Beams, San Diego, 20-24 June 1994, Vol. 2, p. 833.

${ }^{4}$ S. D. Kovaleski, R. M. Gilgenbach, L. K. Ang, Y. Y. Lau, and J. S. Lash, Appl. Surf. Sci. 127-129, 947 (1998).

${ }^{5}$ Th. Witke, A. Lenk, B. Schultrich, and C. Schultheiss, Surf. Coat. Technol. 74-75, 580 (1995).

${ }^{6}$ R. M. Gilgenbach and P. L. G. Ventzek, Appl. Phys. Lett. 58, 1597 (1991).

${ }^{7}$ R. A. Lindley, R. M. Gilgenbach, C. H. Ching, and J. S. Lash, J. Appl. Phys. 76, 5457 (1994). 